\title{
Effects of Different Heave Motion Components on Pilot Pitch Control Behavior
}

\author{
Peter M.T. Zaal* \\ San Jose State University \\ NASA Ames Research Center \\ Moffett Field, CA
}

\author{
Melinda A. Zavala ${ }^{\dagger}$ \\ San Jose State University \\ NASA Ames Research Center \\ Moffett Field, CA
}

\begin{abstract}
The study described in this paper had two objectives. The first objective was to investigate if a different weighting of heave motion components decomposed at the center of gravity, allowing for a higher fidelity of individual components, would result in pilot manual pitch control behavior and performance closer to that observed with full aircraft motion. The second objective was to investigate if decomposing the heave components at the aircraft's instantaneous center of rotation rather than at the center of gravity could result in additional improvements in heave motion fidelity. Twenty-one general aviation pilots performed a pitch attitude control task in an experiment conducted on the Vertical Motion Simulator at NASA Ames under different hexapod motion conditions. The large motion capability of the Vertical Motion Simulator also allowed for a full aircraft motion condition, which served as a baseline. The controlled dynamics were of a transport category aircraft trimmed close to the stall point. When the ratio of center of gravity pitch heave to center of gravity heave increased in the hexapod motion conditions, pilot manual control behavior and performance became increasingly more similar to what is observed with full aircraft motion. Pilot visual and motion gains significantly increased, while the visual lead time constant decreased. The pilot visual and motion time delays remained approximately constant and decreased, respectively. The neuromuscular damping and frequency both decreased, with their values more similar to what is observed with real aircraft motion when there was an equal weighting of the heave of the center of gravity and heave due to rotations about the center of gravity. In terms of openloop performance, the disturbance and target crossover frequency increased and decreased, respectively, and their corresponding phase margins remained constant and increased, respectively. The decomposition point of the heave components only had limited effects on pilot manual control behavior and performance.
\end{abstract}

\section{Nomenclature}

$\begin{array}{ll}A_{d} & \text { disturbance amplitude, deg } \\ A_{t} & \text { target amplitude, deg } \\ a_{z} & \text { heave acceleration, } \mathrm{ft} \mathrm{s}^{-2} \\ a_{z_{\theta}} & \text { pitch heave acceleration, } \mathrm{ft} \mathrm{s}^{-2} \\ e & \text { error signal, deg } \\ f_{d} & \text { disturbance forcing function, deg } \\ f_{t} & \text { target forcing function, deg } \\ H_{\theta} & \text { aircraft pitch response } \\ H_{m f} & \text { simulator motion response } \\ H_{o l, d} & \text { disturbance open-loop response } \\ H_{o l, t} & \text { target open-loop response } \\ H_{p} & \text { pilot response } \\ H_{a_{z}} & \text { aircraft heave response } \\ H_{a_{z_{\theta}}} & \text { aircraft pitch heave response } \\ K_{a_{z}} & \text { heave gain, }- \\ K_{a_{\theta}} & \text { pitch heave gain, }- \\ K_{m} & \text { pilot motion gain, }-\end{array}$

$\begin{array}{ll}K_{m f} & \text { motion filter gain, }- \\ K_{v} & \text { pilot visual gain, }- \\ K_{s} & \text { stick gain, }- \\ k & \text { sinusoid index } \\ l_{x} & \text { longitudinal distance, } \mathrm{ft} \\ m r & \text { motion rating, } \% \\ N_{d} & \text { number of disturbance sine waves } \\ N_{t} & \text { number of target sine waves } \\ n & \text { pilot remnant, deg } \\ n_{d} & \text { disturbance sinusoid frequency integer factor } \\ n_{t} & \text { target sinusoid frequency integer factor } \\ s & \text { Laplace operator } \\ t & \text { time, s } \\ T_{L} & \text { pilot lead time constant, } \mathrm{s} \\ T_{m} & \text { measurement time, } \mathrm{s} \\ u & \text { pilot control input, deg }\end{array}$

${ }^{*}$ Senior Research Engineer, Human Systems Integration Division, NASA Ames Research Center, P.O. Box 1, Moffett Field, CA, 94035 / Mail Stop 262-2; peter.m.t.zaal@ nasa.gov. Member.

${ }^{\dagger}$ Research Associate, Human Systems Integration Division, NASA Ames Research Center, P.O. Box 1, Moffett Field, CA, 94035 / Mail Stop 262-2; melinda.a.zavala@nasa.gov. 


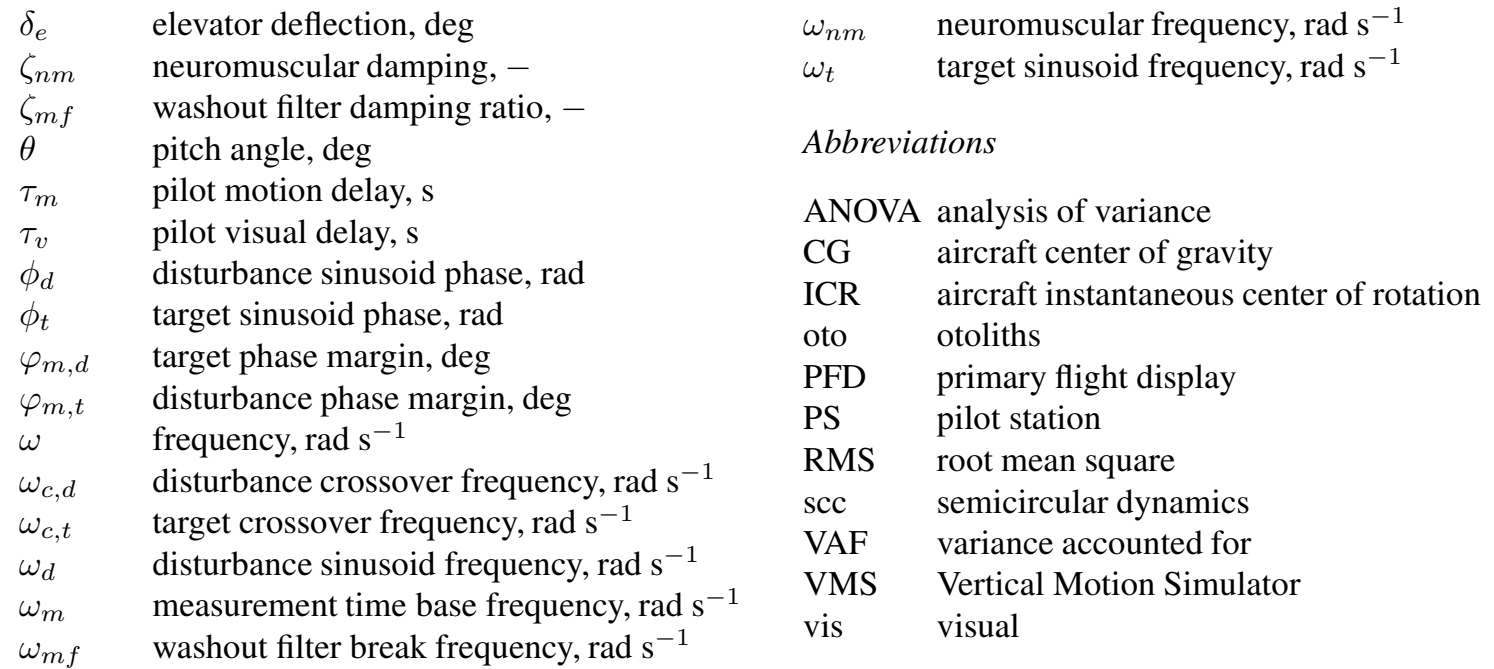

\section{Introduction}

During an aircraft pitch maneuver, the heave accelerations at the pilot station are a superposition of the heave accelerations of the center of gravity and the heave accelerations due to pitch rotations about the center of gravity. In current flight simulators, the total pilot station heave accelerations are attenuated by the motion algorithm; however, the two components of the pilot station heave are very different in nature, and may aid pilots differently depending on the flight task. This paper investigates if a different weighting of the two heave motion components, allowing for optimized motion fidelity of individual components, can result in pilot control behavior and performance more similar to what is observed in real flight. In addition, this paper investigates if using the aircraft's instantaneous center of rotation as the decomposition point for heave motion could result in additional benefits for improving heave motion fidelity during a pitch maneuver.

Considerable research has been performed to investigate how pilot manual control behavior and performance are affected by the different motion components during a pitch attitude control task. Previous research found that the heave accelerations due to pitch rotations have a highly similar effect on control behavior and performance as pitch rotations themselves. ${ }^{1}$ Center of gravity heave was found to have limited effects. A follow-up study found that pilot control behavior and performance are significantly affected when heave motion is filtered or attenuated with a gain. ${ }^{2}$ In Ref. 3 pilot control behavior and performance in a hexapod motion simulator under different pitch and heave motion settings was compared against what was observed in real flight. This study found that control behavior and performance in a simulator motion condition with full pitch motion and filtered heave motion was most similar to what was found in the real aircraft. The experiment in Ref. 4 evaluated how pilot pitch attitude tracking behavior and performance are affected by variations of rotational pitch and translational heave motion settings for a large transport category aircraft. In this study, pilots used the simulator motion more effectively in the heave motion condition with the highest motion filter gains. Furthermore, the addition of rotational pitch motion was found to result in a further increase in pilots' reliance on simulator motion, although the effect was less pronounced compared to findings for smaller aircraft.

The experiment described in this paper adds to the findings from previous studies by considering different weightings of the two types of heave motion, allowing for improved motion fidelity of the two heave components separately in different conditions. Furthermore, the current study used a transport category aircraft model trimmed close to the stall point, whereas previous studies used a model of a small business jet with the pilot station closer to the center of gravity or a large transport category aircraft in a final approach. Finally, the current study used the Vertical Motion Simulator at NASA Ames Research Center, allowing pilots to perform the task with full aircraft motion, the baseline condition.

The paper is structured as follows. First, a brief description of the heave components that make up the total pilot station heave is given. A description of the pitch attitude control task follows. Then, the paper describes the experimental design, which used the Vertical Motion Simulator to test seven hexapod motion conditions and a full aircraft motion condition. After presentation of the results, a discussion follows. The paper ends with conclusions and references. 


\section{Pilot Station Heave Motion}

The control task considered in this study is a pitch attitude control task. Fig. 1 provides a schematic overview of the heave motion components at the center of gravity (CG), instantaneous center of rotation (ICR), and pilot station (PS) of an aircraft in a pitch maneuver. During a change in pitch attitude $\theta$, a pilot at the pilot station experiences rotational accelerations $\ddot{\theta}$ and heave accelerations $a_{z_{P S}}$.

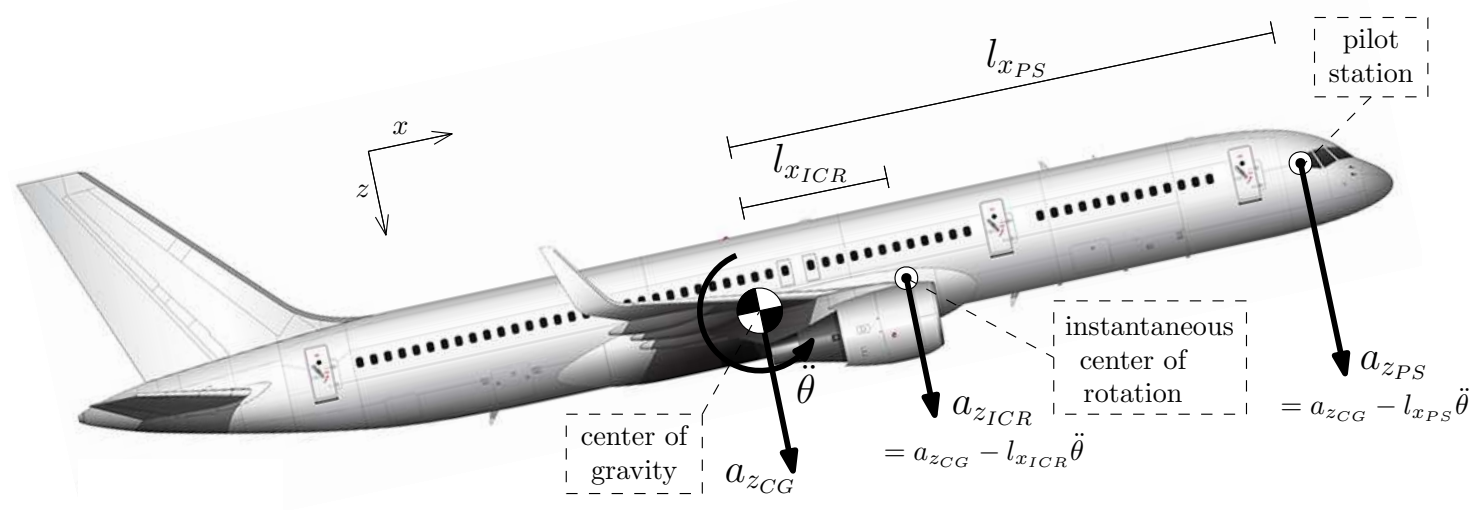

Figure 1. Aircraft heave motion during a pitch maneuver.

\section{II.A. Components with Respect to the Center of Gravity}

In the simulation of aircraft dynamics, forces, moments, and resulting accelerations are typically resolved relative to the CG. Heave accelerations at the PS are a combination of heave accelerations of the CG, $a_{z_{C G}}$, and heave accelerations due to rotations about the CG, $a_{z_{\theta, C G}}$. The CG heave accelerations are a result of variations in lift due to changes in angle of attack. The total heave acceleration at the pilot station is then given by:

$$
a_{z_{P S}}=a_{z_{C G}}+a_{z_{\theta, C G}}=a_{z_{C G}}-l_{x_{P S}} \ddot{\theta}
$$

with $l_{x_{P S}}$ the longitudinal distance between the center of gravity and the pilot station, with a value of $48 \mathrm{ft}$ for the aircraft simulated in this study. Similarly, a pilot experiences surge accelerations at the pilot station during a pitch maneuver, which are a combination of surge accelerations of the CG and surge accelerations due to rotations about the CG. The surge accelerations have been omitted from Fig. 1 for brevity, but were simulated in the experiment.

During an aircraft pitch attitude control task, pilots receive additional feedback of the aircraft pitch and heave motion components, allowing them to close extra feedback loops around the controlled aircraft dynamics and increase performance. Both pitch accelerations $\ddot{\theta}$ and pitch heave accelerations with respect to the CG $a_{z_{\theta, C G}}$ are directly correlated with aircraft pitch. However, the CG heave $a_{z_{C G}}$ has a less direct relation to pitch due to the slower altitude mode of the aircraft dynamics. A previous study showed that this heave component does not significantly affect pitch control behavior and performance, while it requires the most simulator motion. ${ }^{1}$ The goal of the experiment described here is to investigate how different weightings of CG heave and pitch heave with respect to the CG affect pitch control behavior and performance.

\section{II.B. Components with Respect to the Instantaneous Center of Rotation}

The characteristics of the instantaneous center of rotation are explored in this experiment to investigate if pilot station heave decomposed with respect to this point provides alternative ways to increase heave motion cueing fidelity in flight simulators. The ICR is located along the longitudinal axis of the aircraft, like the center of gravity, however differs in that it's location is fore or aft of the CG and remains on the original flight path as the aircraft rotates about its CG. The ICR location is typically in front of the CG for conventional long-aft tailed aircraft, such as the aircraft considered in this study. In Fig. 1, the longitudinal distance between CG and ICR, $l_{x_{I C R}}$, is $10.3151 \mathrm{ft}$, and the longitudinal distance 
between the pilot station and the ICR is $37.6849 \mathrm{ft}$. Similarly to decomposing the heave with respect to the CG, the total heave acceleration at the pilot station decomposed with respect to the ICR is given by:

$$
a_{z_{P S}}=a_{z_{I C R}}+a_{z_{\theta, I C R}}=a_{z_{I C R}}-\left(l_{x_{P S}}-l_{x_{I C R}}\right) \ddot{\theta}
$$

At the ICR, the vertical acceleration due to the change in aerodynamic lift is just balanced by that due to the pitch heave acceleration with respect to the CG. As such, the ICR is of particular interest for this experiment since it does not have net instantaneous vertical acceleration after an elevator control input, whereas the CG has a vertical acceleration opposite of the direction of pitch for the aircraft considered here. Fig. $2 b$ provides the aircraft pitch acceleration response and Fig. 2c the heave acceleration responses to the elevator sine input depicted in Fig. 2a. Note that a negative elevator input results in a positive or nose-up pitch response and a negative pitch heave response $\left(-l_{x_{P S}} \ddot{\theta}\right)$.

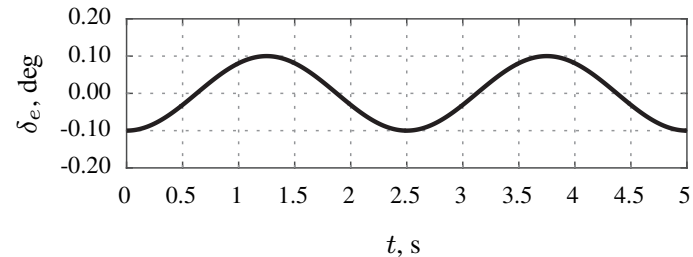

(a) Elevator input

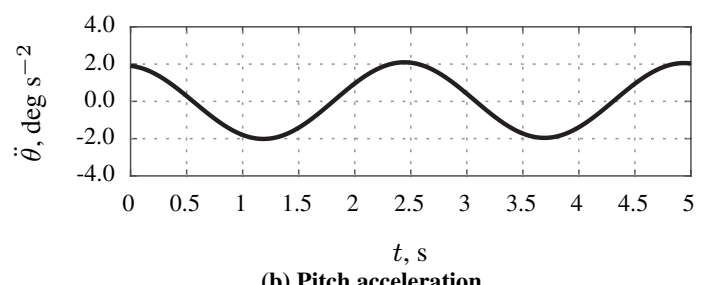

(b) Pitch acceleration

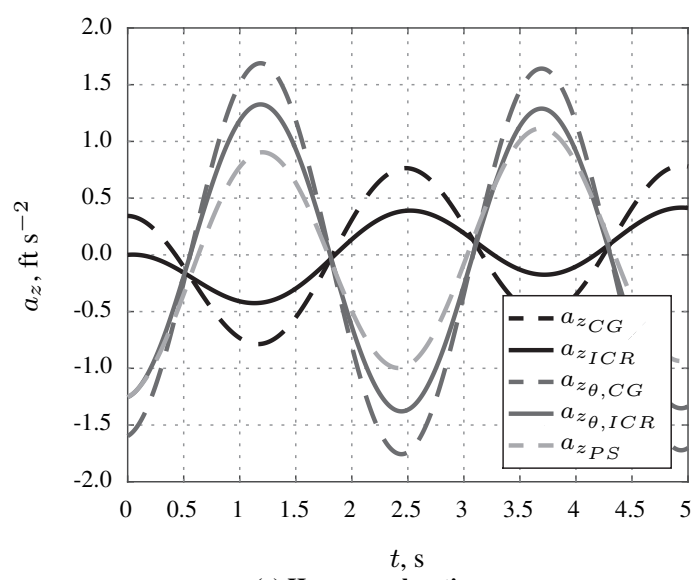

(c) Heave acceleration

Figure 2. Aircraft pitch and heave responses to an elevator sine input.

Fig. 2c shows that the instantaneous CG heave after an elevator input is opposite of the direction of pitch. The instantaneous ICR heave is zero. However, the steady state response of both is opposite the direction of pitch. The pitch heave with respect to the CG has a larger magnitude compared to the pitch heave with respect to the ICR due to the longer distance between the PS and the CG than the PS and the ICR. Finally, the total instantaneous PS heave is equivalent to the instantaneous pitch heave with respect to the ICR and lower in magnitude compared to the pitch heave with respect to the CG. Note that summing the two heave components resolved at the CG results in the same PS heave as summing the two heave components resolved at the ICR if both components have a weighting of one.

\section{Pitch Attitude Control Task}

\section{III.A. Control Diagram}

Fig. 3 depicts the pitch control task performed by pilots in the VMS. Pilots' task was to actively minimize the pitch error, $e$, presented on a compensatory display, which resembled a basic Primary Flight Display (PFD). Pilots acted on the controlled pitch dynamics, $H_{\theta}$, by making control inputs, $u$, with a sidestick. The sidestick used a gain $K_{s}=0.8$. In addition to the visual stimuli presented on the display, pitch and heave motion were provided by the simulator motion logic with dynamics $H_{m_{\theta}}$ and $H_{m_{a z}}$, respectively. The level of pitch rotational motion feedback pilots received was constant. However, the level of heave motion feedback varied according to the motion conditions presented in Section IV.A.1. Heave motion was a summation of the heave motion at the CG or ICR $\left(H_{a_{z_{C G}}}\right.$ or $\left.H_{a_{z_{I C R}}}\right)$ and the pitch heave motion with respect to the CG or ICR $\left(H_{a_{z_{\theta, C G}}}\right.$ or $\left.H_{a_{z_{\theta} I C R}}\right)$. The weighting of the two components was determined by their respective gains $\left(K_{a_{z_{C G}}}, K_{a_{z_{I C R}}}, K_{a_{z_{\theta, C G}}}\right.$, and $\left.K_{a_{z_{\theta, I C R}}}\right)$, see Section IV.A.1.

In the control task of Fig. 3, a pilot can be modeled by linear response functions. ${ }^{5}$ A remnant signal, $n$, accounts for measurement noise and nonlinearities not captured by these response functions. In order to separate pilots' visual and motion responses, a disturbance forcing function, $f_{d}$, was added to the controlled element output, feeding directly into the display and VMS motion system. A target forcing function, $f_{t}$, feeds into the display only through the error, $e$, the difference between $f_{t}$ and the disturbed pitch attitude, $\theta$. 


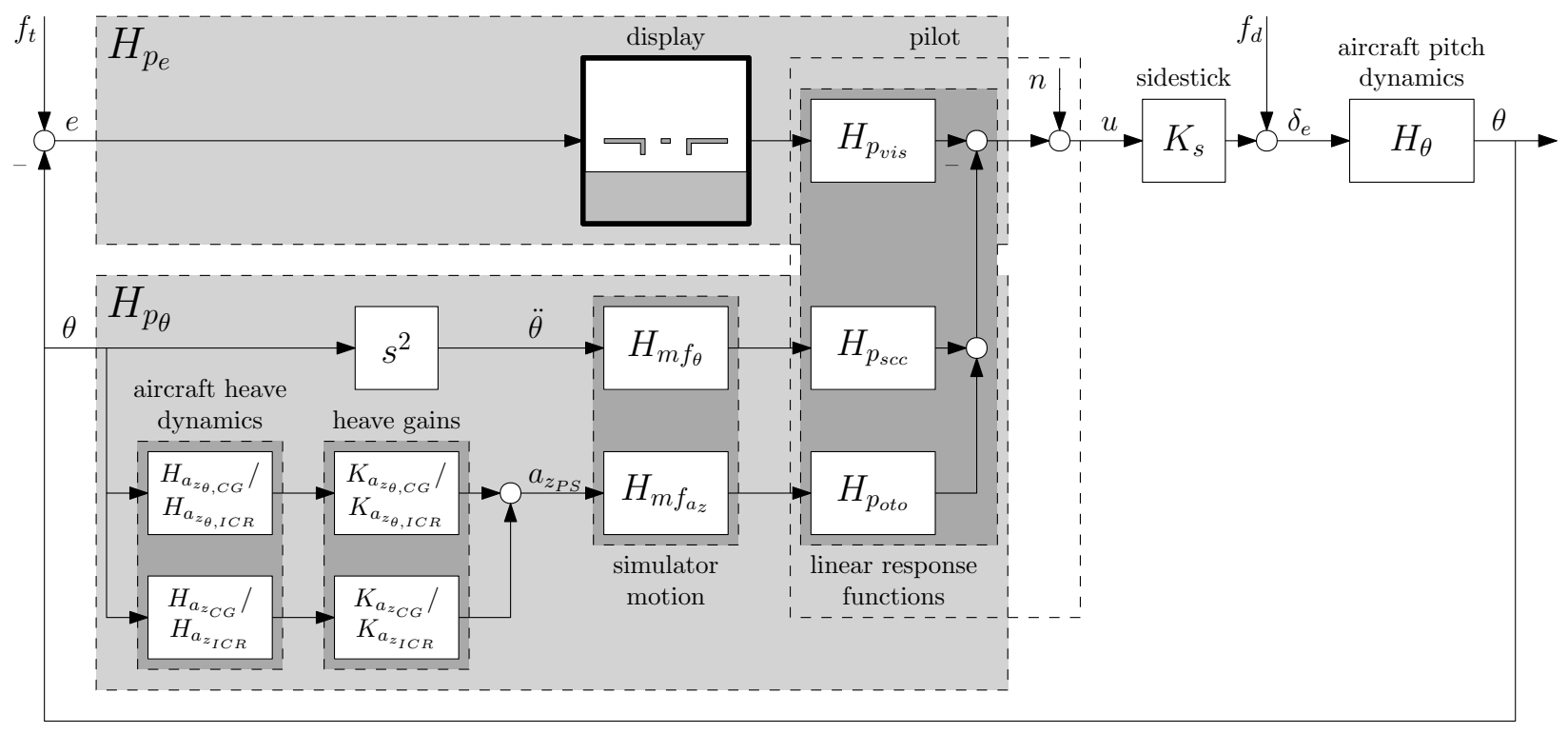

Figure 3. Pitch attitude control Task.

The pilot in Fig. 3 should idealy be modeled by a linear function characterizing the response to visual cues $H_{p_{v i s}}$, a linear function characterizing the response to rotational motion cues through the semicircular canals $H_{p_{s c c}}$, and a linear function characterizing the response to translational motion cues through the otoliths $H_{p_{\text {oto }}}$. However, the two forcing functions $f_{d}$ and $f_{t}$ only allow for the identification of two pilot response functions. ${ }^{6}$ In this study we choose to use the visual error signal $e$, the aircraft pitch attitude $\theta$, and the pilot control input $u$ to identify the pilot. This allows for the identification of a visual response function $H_{p_{e}}$ and a motion response function $H_{p_{\theta}}$. Note that this motion response function is a lumped response of the responses to rotational motion with the semicircular canals and translational motion motion with the otoliths. Also note that, using this approach, the aircraft heave dynamics and gains, and the simulator motion dynamics are part of the pilot motion response function.

\section{III.B. Controlled Aircraft Dynamics}

The simulated aircraft dynamics were of a mid-sized twin-engine commercial transport aircraft, with a gross weight of 185,800 lbs. The aircraft model's dynamics were linearized close to the stall point, at an altitude of 41,000 ft and an airspeed of $150 \mathrm{kts}$. The controlled pitch dynamics were defined by (Fig. 3):

$$
H_{\theta}=\frac{\theta}{\delta_{e}}=\frac{28.4474\left(346.5 s^{2}+32.03 s+1\right)}{\left(245.6 s^{2}-3.409 s+1\right)\left(2.105 s^{2}+0.9387 s+1\right)}
$$

The CG heave response to pitch variations was defined by:

$$
H_{a_{z_{C G}}}=\frac{a_{z_{C G}}}{\theta}=\frac{10.315 s(s+2.506)(s-2.148)(s-0.01235)}{\left(s^{2}+0.09244 s+0.002886\right)}
$$

and the CG pitch heave response to pitch variations was defined by:

$$
H_{a_{z_{\theta, C G}}}=\frac{a_{z_{\theta, C G}}}{\theta}=-48 s^{2}
$$

The heave components decomposed at the ICR were defined by:

$$
H_{a_{z_{I C R}}}=\frac{a_{z_{I C R}}}{\theta}=\frac{2.6054 s(s-21.33)(s-0.01234)}{\left(s^{2}+0.09244 s+0.002886\right)}
$$

and:

$$
H_{a_{z_{\theta, I C R}}}=\frac{a_{z_{\theta, I C R}}}{\theta}=-37.685 s^{2}
$$


Surge motion was also simulated during the experiment. The surge transfer functions equivalent to Eqs. 4 to 7 are omitted here for brevity.

\section{III.C. Simulator Motion Logic}

The VMS motion logic consists of second-order high-pass washout filters to attenuate the rotational and translational aircraft model accelerations:

$$
H_{m f_{\theta}, m f_{a_{z}}}(s)=K_{m f_{\theta}, m f_{a_{z}}} \frac{s^{2}}{s^{2}+2 \zeta_{m f_{\theta}, m f_{a_{z}}} \omega_{m f_{\theta}, m f_{a_{z}}} s+\omega_{m f_{\theta}, m f_{a_{z}}}^{2}}
$$

where $K_{m f_{\theta}, m f_{a_{z}}}$ is the motion filter gain. $\zeta_{m f_{\theta}, m f_{a_{z}}}$ and $\omega_{m f_{\theta}, m f_{a_{z}}}$ are the washout damping ratio and break frequency, respectively. Pitch motion was present in all experimental conditions. The gain of the pitch motion $K_{m f_{\theta}}$ was 1.00 and the pitch washout break frequency $\omega_{m f_{\theta}}$ was $0.10 \mathrm{rad} / \mathrm{s}$. The heave motion gain $K_{m f_{a_{z}}}$ and heave washout break frequency $\omega_{m f_{a_{z}}}$ varied depending on the experimental condition (see Section IV.A.1). Surge motion was supplied with motion settings consistent with the heave motion settings. All damping ratios were set to 0.707.

\section{III.D. Pilot Model}

In order to characterize pilot control behavior for each pilot, linear transfer functions were used to describe the visual and motion responses. Nonlinear behavior and measurement noise are captured by the remnant signal $n$ (see Fig. 3). According to the crossover model theorem, a human operator adjusts his or her control behavior to the controlled dynamics such that the pilot-vehicle open-loop response approximates a single integrator near the crossover frequency. ${ }^{5}$

Taking into account the need for visual lead generation at higher frequencies due to the characteristics of the controlled dynamics, the visual response transfer function used to model manual control behavior in Fig. 3 is defined by:

$$
H_{p_{e}}(s)=K_{v}\left(1+T_{L} s\right) e^{-\tau_{v} s} \frac{\omega_{n m}^{2}}{s^{2}+2 \zeta_{n m} \omega_{n m} s+\omega_{n m}^{2}}
$$

and the motion response transfer function by:

$$
H_{p_{\theta}}(s)=s K_{m} e^{-\tau_{m} s} \frac{\omega_{n m}^{2}}{s^{2}+2 \zeta_{n m} \omega_{n m} s+\omega_{n m}^{2}}
$$

In this multimodal pilot model representation, pilot equalization is defined by the visual gain $K_{v}$, the visual lead time constant $T_{L}$, and the motion gain $K_{m}$. The pilot's limitations are characterized by the visual delay $\tau_{v}$, the motion delay $\tau_{m}$, and the neuromuscular dynamics with a neuromuscular frequency $\omega_{n m}$ and a neuromuscular damping $\zeta_{n m}$.

It must be noted again that $H_{p \theta}$ represents the sum of multiple separate motion feedback channels defining the use of angular accelerations detected by the semicircular canals, linear accelerations detected by the otoliths, and motion cues from the somatosensory system (Fig. 3). This modeling approach has successfully been used in previous research. ${ }^{2}$ Furthermore, visual display dynamics are included in $H_{p_{e}}$, and the aircraft heave dynamics and simulator motion dynamics are included in and $H_{p_{\theta}}$.

In the frequency domain, pilot performance in attenuating the target and disturbance signals is determined by the crossover frequencies and phase margins of the target and disturbance open-loop dynamics, respectively. ${ }^{7}$ Using the control task diagram in Fig. 3 and the pilot response functions in Eqs. 9 and 10, the disturbance and target open-loop responses are given by:

$$
\begin{gathered}
H_{o l, d}(s)=\left[H_{p_{e}}(s)+H_{p_{\theta}}(s)\right] K_{s} H_{\theta}(s) \\
H_{o l, t}(s)=\frac{H_{p_{e}}(s) K_{s} H_{\theta}(s)}{1+H_{p_{\theta}}(s) K_{s} H_{\theta}(s)}
\end{gathered}
$$

The disturbance and target crossover frequencies, $\omega_{c, d}$ and $\omega_{c, t}$, are the frequencies where the magnitude of the disturbance and target open-loop responses is 1.0. The corresponding phase margins, $\varphi_{m, d}$ and $\varphi_{m, t}$, are the phase differences from -180 degrees at these crossover frequencies. 


\section{III.E. Forcing Functions}

The target and disturbance forcing functions were sum-of-sines signals defined by:

$$
f_{d, t}(t)=\sum_{k=1}^{N_{d, t}} A_{d, t}(k) \sin \left[\omega_{d, t}(k) t+\phi_{d, t}(k)\right]
$$

where $A_{d, t}(k), \omega_{d, t}(k)$, and $\phi_{d, t}(k)$, respectively, indicate the amplitude, frequency, and phase of the $k^{t h}$ sine in $f_{d}$ or $f_{t}$. $N_{d, t}$ represents the number of sine waves. Subscripts $d$ and $t$ are the disturbance and target forcing function, respectively. Both $f_{d}$ and $f_{t}$ consist of ten individual sinusoids with a different amplitude, frequency, and phase. A summary of all forcing function properties can be found in Table 1.

Table 1. Forcing function properties.

\begin{tabular}{|c|c|c|c|c|c|c|c|}
\hline \multicolumn{4}{|c|}{ target, $f_{t}$} & \multicolumn{4}{|c|}{ disturbance, $f_{d}$} \\
\hline$n_{t},-$ & $\omega_{t}, \operatorname{rad} s^{-1}$ & $A_{t}, \operatorname{deg}$ & $\phi_{t}, \mathrm{rad}$ & $n_{d},-$ & $\omega_{d}, \operatorname{rad} s^{-1}$ & $A_{d}, \mathrm{deg}$ & $\phi_{d}, \mathrm{rad}$ \\
\hline 2 & 0.1534 & 0.0105 & 0.1355 & 3 & 0.2301 & 0.5818 & -1.4796 \\
\hline 5 & 0.3835 & 0.0098 & -0.1664 & 6 & 0.4602 & 0.5306 & -0.0745 \\
\hline 11 & 0.8437 & 0.0091 & 2.9016 & 13 & 0.9971 & 0.3711 & 0.7006 \\
\hline 23 & 1.7641 & 0.0283 & 5.6383 & 27 & 2.0709 & 0.1674 & -1.9563 \\
\hline 37 & 2.8379 & 0.0403 & 2.8648 & 41 & 3.1447 & 0.0901 & -2.8131 \\
\hline 51 & 3.9117 & 0.0477 & 4.8718 & 53 & 4.0650 & 0.0605 & 2.1026 \\
\hline 71 & 5.4456 & 0.0569 & 1.0245 & 73 & 5.5990 & 0.0375 & -2.6178 \\
\hline 101 & 7.7466 & 0.0725 & 5.0337 & 103 & 7.9000 & 0.0238 & 2.2550 \\
\hline 137 & 10.5078 & 0.0967 & 4.1487 & 139 & 10.6612 & 0.0174 & -0.6739 \\
\hline 191 & 14.6495 & 0.1458 & 0.4274 & 194 & 14.8796 & 0.0135 & 0.1942 \\
\hline
\end{tabular}

The frequencies of the sinusoids, $\omega_{d}(k)$ and $\omega_{t}(k)$, were all integer multiples of the measurement time base frequency, $\omega_{m}=2 \pi / T_{m}=0.0767 \mathrm{rad} / \mathrm{s}$, where $T_{m}=81.92 \mathrm{~s}$ is the measurement time. The selected integer multiples were used in a number of earlier experiments and ensured that the ten sinusoid frequencies in each signal covered the frequency range of human control at regular intervals on a logarithmic scale.

A second-order low-pass filter was used to determine the amplitudes of the individual sines for both the target and the disturbance forcing functions. ${ }^{1}$ This second-order filter reduced the magnitude of the amplitudes at the higher frequencies, yielding a tracking task that is not overly difficult. The amplitude distributions $A_{t}(k)$ and $A_{d}(k)$ were scaled to obtain a variance for $f_{t}$ of $0.4 \mathrm{deg}^{2}$, and a resulting variance for $f_{d}$ in the pitch response of $0.4 \mathrm{deg}^{2}$. This relative strength of the target and disturbance signals, and the amplitude variation determined by the second-order filter were successfully applied in previous experiments. ${ }^{8,9}$

To determine the forcing function phase distributions, numerous random sets of phases were generated. Two sets of phases were chosen for the target and disturbance forcing functions that yielded signals with a Gaussian-like distribution and an average crest factor. ${ }^{10}$

\section{Experimental Design}

\section{IV.A. Method}

\section{IV.A.1. Experimental Conditions}

The independent variable manipulated in the experiment was the heave motion at the pilot station. Table 2 provides an overview of the eight motion conditions in the experiment. Conditions 1 through 5 used heave motion components decomposed at the CG. Conditions 6 and 7 used heave motion components decomposed at the ICR. The motion in conditions 1 through 7 was tuned such that it remained inside the motion envelope of a typical hexapod motion system with 60-inch legs. Condition 8 was a full-motion condition that used as much of the VMS motion space as possible. This condition represented the full aircraft motion and served as a baseline. In each condition, surge motion congruent with the heave motion was supplied; that is, with the same decomposition point, gains, and washout parameters. Full pitch rotational motion was present in all conditions.

In conditions 1 through 5 the weighting of the CG heave and pitch heave with respect to the CG was varied by manipulating their respective gains. C1 provided CG heave only, while C5 provided pitch heave with respect to the CG only. C6 and C7 were identical to C1 and C5, respectively, but with the ICR as the decomposition point. C8 
Table 2. Experimental Conditions.

\begin{tabular}{cccccccc}
\hline \hline Condition & $\begin{array}{c}\text { CG Pitch-Heave } \\
\text { Gain }\end{array}$ & CG Heave Gain & $\begin{array}{c}\text { ICR Pitch-Heave } \\
\text { Gain }\end{array}$ & ICR Heave Gain & $\begin{array}{c}\text { Motion } \\
\text { Washout Gain }\end{array}$ & $\begin{array}{c}\text { Washout Break } \\
\text { Frequency }\end{array}$ & Motion Limits \\
\cline { 2 - 7 } & $K_{a_{z_{\theta}, C G}}$ & $K_{a_{z_{C G}}}$ & $K_{a_{z_{\theta, I C R}}}$ & $K_{a_{z} I C R}$ & $K_{m f_{a_{z}}}$ & $\omega_{m f_{z}}$ & \\
\hline $\mathrm{C} 1$ & 0.00 & 1.00 & - & - & 0.60 & 0.60 & hexapod \\
$\mathrm{C} 2$ & 0.25 & 0.75 & - & - & 0.70 & 0.50 & hexapod \\
$\mathrm{C} 3$ & 0.50 & 0.50 & - & - & 0.80 & 0.40 & hexapod \\
$\mathrm{C} 4$ & 0.75 & 0.25 & - & - & 0.90 & 0.30 & hexapod \\
$\mathrm{C} 5$ & 1.00 & 0.00 & - & - & 1.00 & 0.20 & hexapod \\
$\mathrm{C} 6$ & - & - & 0.00 & 1.00 & 0.60 & 0.60 & hexapod \\
$\mathrm{C} 7$ & - & - & 1.00 & 0.00 & 1.00 & 0.20 & hexapod \\
$\mathrm{C} 8$ & 1.00 & 1.00 & - & - & 1.00 & 0.20 & VMS \\
\hline \hline
\end{tabular}

was the only condition with full CG heave and pitch heave with respect to the CG. Note that decomposing the heave motion at the ICR for C8 would result in exactly the same motion.

Table 2 also provides the motion gains and washout break frequencies for each motion condition. For C1, with CG heave only, a relatively low motion gain of 0.60 and high break frequency of 0.60 were required to keep the motion within the hexapod motion envelope. Going from $\mathrm{C} 1$ to $\mathrm{C} 5$, with progressively less CG heave, less attenuation of the motion was required. For C5, with CG pitch heave only, hardly any motion attenuation was required; that is, the motion gain was 1.00 and the break frequency was 0.20 . C6 and C7 used the same motion parameters as $\mathrm{C} 1$ and C5 respectively, to allow for a direct comparison of results. Finally, for the full-motion condition C8 motion washout parameters equivalent to $\mathrm{C} 5$ were used. Note that $\mathrm{C} 5$ allows for the same motion washout parameters as the full-motion condition C8 while staying within the much smaller hexapod motion limits by eliminating the CG heave component from the pilot-station heave motion.

The conditions of Table 2 allow for two separate analyses of variance (ANOVAs) to be performed. In the first ANOVA, the effect of different weightings of the heave components decomposed with respect to the CG on pilot control behavior and performance can be investigated using conditions $\mathrm{C} 1$ through $\mathrm{C} 5$ and $\mathrm{C} 8$, with $\mathrm{C} 8$ as the baseline or reference condition. In this analysis, the factor is the heave component (HC6) with six levels. Next, the effect of the decomposition point (CG or ICR) and the heave component (CG/ICR heave or pitch heave with respect to the $\mathrm{CG} / \mathrm{ICR}$ ) can be investigated in a two-way ANOVA. In this analysis, the factors are the decomposition point and the heave component, both with two levels (DP2 and HC2).

\section{IV.A.2. Apparatus}

This experiment used the VMS with the rotorcraft cab (R-CAB), see Fig. $4 .{ }^{11}$ A summary of VMS capabilities can be found in Ref. 12. Pilots were seated on a chair positioned in the center of the cab and controlled the pitch attitude through a joystick located on the right side of the seat as seen in Fig. 5. Pilots had a simplified PFD in front of them that displayed the pitch error (see Fig. 6). No out-the-window view was projected. The simulator was configured to use the long horizontal motion axis for surge motion, instead of the normal configuration in which the long axis is used for sway motion. A Microsoft ${ }^{\circledR}$ Surface $^{\mathrm{TM}}$ tablet was used by pilots to provide motion ratings.

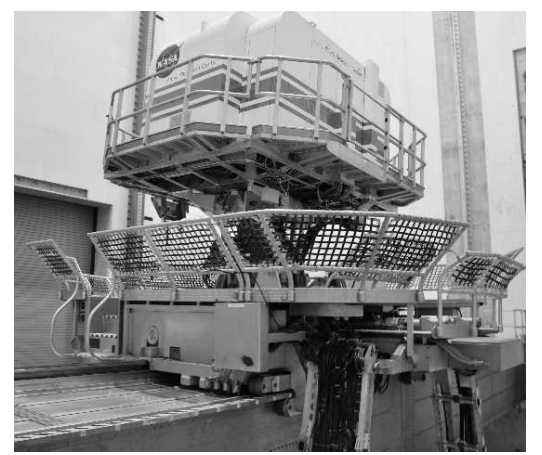

Figure 4. Vertical Motion Simulator.

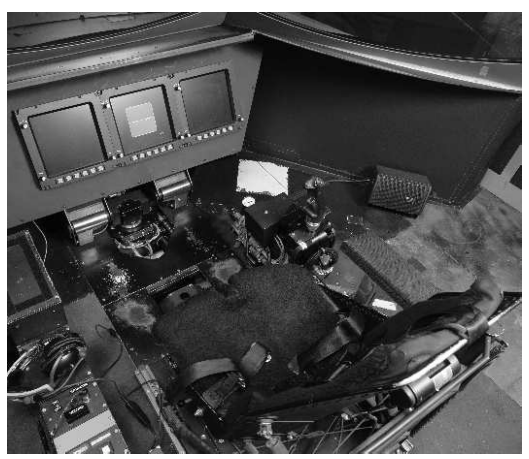

Figure 5. Cockpit setup.

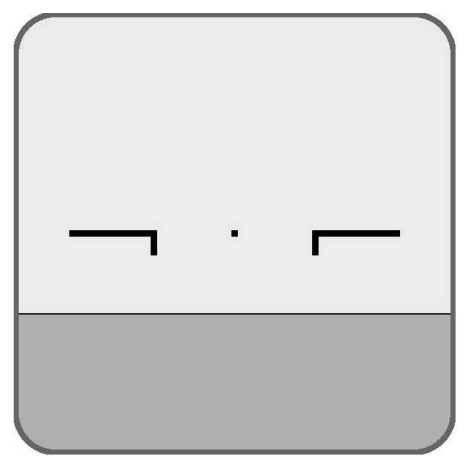

Figure 6. Primary flight display. 


\section{IV.A.3. Participants}

Twenty-one general aviation pilots participated in the experiment. Three pilots had 1,500 or more hours of flying. Two pilots had significantly more flight hours than the rest: 3,200 and 5,000 hours. The average number of flight hours was 897 with a standard deviation of $\pm 1,173$. Most pilots had fixed-base simulator experience, with an average of 85 hours and standard deviation of \pm 128 . Pilots fell between the ages of 21 and 64, with the majority under 40 years with an average age of 30.9 years and a standard deviation of \pm 10.8 years.

\section{IV.A.4. Procedures}

Before the start of the experiment, each pilot received a thorough briefing informing them of the purpose of the experiment and overall procedures, including the goal of the task and suggestions on how to compensate for the disturbances more effectively by informing them with examples of good and bad control inputs on paper. In addition, pilots were instructed on how to use a sliding scale on a Microsoft ${ }^{\circledR}$ Surface ${ }^{\mathrm{TM}}$ tablet to rate the fidelity of the simulator motion in each run against the baseline full-motion condition. Pilots were only given detail about the full-motion condition; and that it was present between other motion conditions throughout the experiment. No specific details about the other motion conditions were provided. To motivate pilots to improve their performance, they were told the current best performance score and encouraged to improve on it.

Each pilot performed 64, 90-second runs, in two sessions of 24 runs and one session of 16 runs with a ten-minute break between sessions. During and between each session, pilots were allowed to take longer breaks if they requested. The first session was used for training. This session began with the baseline full-motion condition C8 performed eight times, followed by 16 runs with the eight motion conditions randomly varied. The eight baseline condition runs at the start of the session enabled pilots to more accurately rate the other motion conditions against this baseline condition. The eight motion conditions were randomly varied for the second and third sessions. Five runs per condition from these sessions were considered measurement runs and were used to calculate the results.

At the end of each run, the root mean square (RMS) of the pitch error appeared at the bottom right of the PFD to give pilots feedback on their performance. The smaller the RMS value, the better performance they achieved throughout that run. Pilots were encouraged to always try to improve their performance.

\section{IV.A.5. Dependent Measures}

One subjective dependent measure and 14 objective dependent measures were analyzed. Motion ratings $m r$ were used to gain insight into pilots' subjective opinion about the motion fidelity of each motion condition. Pilots used a visual analogue fidelity scale to rate the motion fidelity between $0 \%$ and $100 \%$ after each run. Zero percent meant the motion had low fidelity and was not comparable to the baseline full-motion condition and $100 \%$ meant the motion had high fidelity and was equivalent to the baseline condition.

Several dependent measures were calculated for every pilot to evaluate how manual control behavior and performance changed between each condition. Pilot control performance and intensity were evaluated in terms of the RMS of the pitch error, $R M S_{e}$, and longitudinal control input, $R M S_{u}$, respectively. These two measures were calculated for every single measurement run and averaged for each condition.

The pilot model defined in Eqs. 9 and 10 contains seven parameters $\left(K_{v}, T_{L}, \tau_{v}, K_{m}, \tau_{m}, \zeta_{n m}\right.$, and $\left.\omega_{n m}\right)$, which were estimated from experiment data using maximum likelihood estimation. Simulation data were averaged over five runs for each condition before proceeding with the parameter estimation in order to reduce the influence of pilot remnant on accuracy of the parameter estimates. First, a genetic algorithm optimization was used to obtain rough initial estimates of the parameters. Following, a Gauss-Newton optimization algorithm obtained the set of parameters that provides the pilot model that best fit the experiment data. ${ }^{13}$ The control signal variance accounted for $(V A F)$ was calculated as a measure of the accuracy of the pilot model in describing the measured control signal data.

Finally, the open-loop responses of Eqs. 11 and 12 were used to calculate the target and disturbance crossover frequencies $\omega_{c, t}$ and $\omega_{c, d}$, and phase margins $\varphi_{m, t}$ and $\varphi_{m, d}$, for each condition.

\section{IV.B. Hypotheses}

Several hypotheses were formulated based on previous research. ${ }^{1-4}$ Due to the direct relation between CG pitch heave and pitch accelerations, and the less direct relation between CG heave and pitch accelerations, it was hypothesized that increasing the ratio of CG pitch heave to CG heave, while increasing motion logic gains and decreasing washout break frequencies, would allow for improvements in pilot performance and significant changes in pilot manual control 
behavior. Increased pilot visual and motion gains and a reduced visual lead time constant were expected with higher ratios of CG pitch heave to CG heave, indicating an increased use of simulator motion cues by pilots. We hypothesized that pilot manual control behavior and performance with higher levels of CG pitch heave would be more similar to what is observed with full aircraft motion. We expected differences between using the CG and ICR as the decomposition point to be small due to the relatively short distance of the ICR from the CG. Condition 5 presents an interesting case: more pitch heave is present in this condition than condition 7 due to the longer distance between the PS and the CG. This may cause the pilot to experience pitch heave motion cues that are exaggerated compared to full aircraft motion, leading to better performance than in the full aircraft motion condition. ${ }^{14}$ For this reason, we hypothesized that pilot control behavior and performance in the condition with ICR pitch heave (C7) or $0.75 \% \mathrm{CG}$ pitch heave (C4) might be most similar to what is observed with full aircraft motion.

\section{Results}

This section presents the combined results of 19 out of the 21 pilots who participated in the experiment. Experiment data from two pilots were not sufficient to generate accurate pilot model parameter estimates. Results from these two pilots were left out of the entire analysis. The figures in this section depict means and $95 \%$ confidence intervals for each condition. Data were corrected for between-subject variability. Condition C8 served as a reference condition. Data from this condition have been marked with a grey horizontal line in each figure.

Two separate repeated-measures ANOVAs were performed on each dependent measure to find significant differences between conditions. A one-way ANOVA was performed to find significant differences introduced by the different weighting of heave motion components decomposed at the CG (C1-C5 and C8). Data from these conditions are provided on the left side of the dashed vertical line in each plot below. The corresponding ANOVA results are given in the appendix in Table 3a. A two-way ANOVA was performed to find significant differences introduced by the decomposition point and the heave components decomposed at these points (C1,C5,C6,C7). Data from these conditions are provided on the right side of the dashed vertical line in each figure. Table $3 \mathrm{~b}$ in the appendix provides the corresponding ANOVA results. More details about the ANOVA assumptions and procedures are provided in the appendix.

\section{V.A. Motion Ratings}

The motion ratings pilots gave on an analogue fidelity scale are provided in Fig. 7. Pilots rated the motion in each condition against the motion in the baseline condition C8 during the first eight runs of the experiment. Zero percent meant the motion was not like the baseline or full aircraft motion at all, while $100 \%$ meant the motion was equivalent.

A significant difference in motion ratings was introduced by the different weightings of the heave motion components decomposed at the CG (left side of Fig. 7). The motion in $\mathrm{C} 8$ was not rated $100 \%$ while it was equivalent to the motion pilots were told to rate against. Motion in $\mathrm{C} 4$ and $\mathrm{C} 5$, with a higher percentage of CG pitch heave compared to $\mathrm{CG}$ heave, was rated as high as the baseline motion. Motion in the remaining conditions

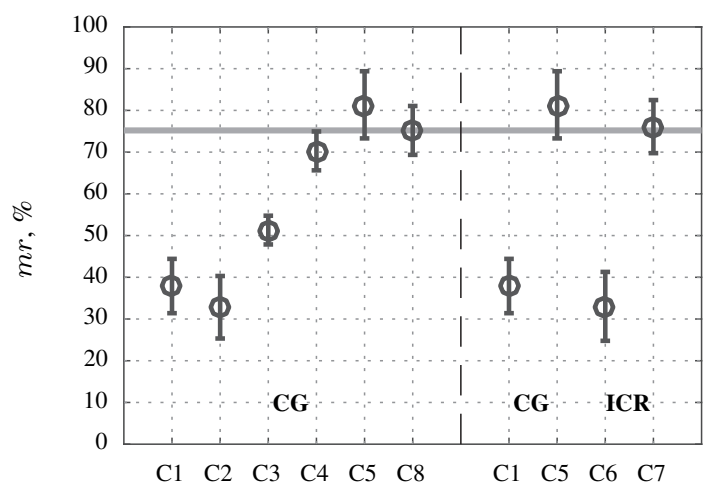

Figure 7. Motion fidelity ratings. was rated significantly lower, with no significant difference between $\mathrm{C} 1$ and $\mathrm{C} 2$.

For C1,C5,C6 and C7 (right side of Fig. 7), no significant interaction was found between the heave decomposition point and the heave component. However, a significant difference was introduced by both factors separately. The motion in the conditions with pitch heave only (C5 and C7) was rated the same as the motion in the reference condition, while motion in the conditions with $\mathrm{CG}$ or ICR heave only ( $\mathrm{C} 1$ and $\mathrm{C} 6)$ was rated significantly lower. The motion in the conditions using the ICR as the decomposition point (C6 and $\mathrm{C} 7$ ) were rated significantly lower compared to using the $\mathrm{CG}$ as the decomposition point ( $\mathrm{C} 1$ and $\mathrm{C} 5$ ). 


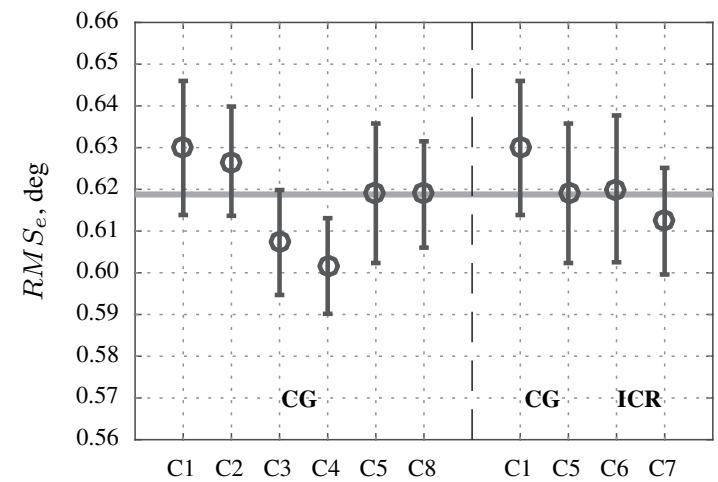

(a) Tracking Performance

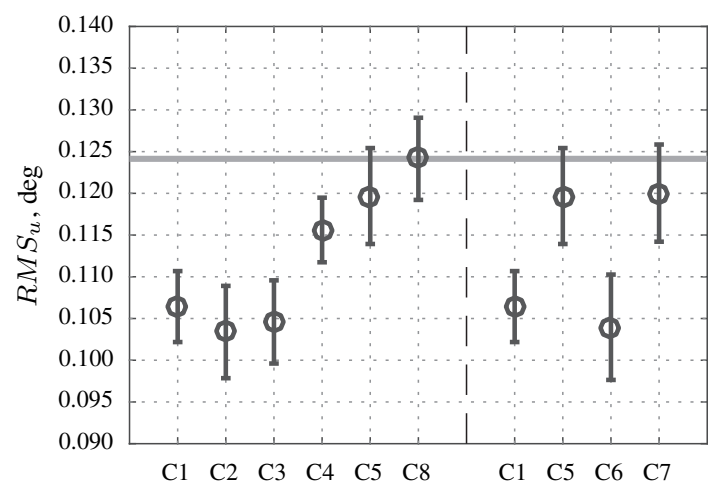

(b) Control Activity

Figure 8. Pilot tracking performance and control activity.

\section{V.B. Performance and Control Activity}

Fig. 8a provides pilot performance data and Fig. 8b control activity data for all conditions. A lower $R M S_{e}$ corresponds to better performance. A marginally significant difference in performance was introduced by the different weightings of heave motion components with respect to the CG. For C3 and C4 performance was better than in the other conditions. Performance in the condition with pitch heave with respect to the CG only (C5) was closest to performance in the baseline condition (C8). No significant differences were introduced by the decomposition point, the heave component, or the interaction between the two.

Pilot control activity was significantly affected by the heave motion components decomposed with respect to the CG. For the conditions with a higher percentage of pitch heave compared to CG heave (C4 and C5), control activity was similar to the baseline condition $\mathrm{C} 8$. In the conditions with a lower or equal percentage of pitch heave compared CG heave (C1-C3), control activity was significantly lower than in the baseline condition. No significant interaction was introduced by the decomposition point and the heave component (DP2 $\times \mathrm{HC} 2$ ), or the decomposition point. However, the heave component (HC2) introduced a significant difference in control activity, similar to the significant difference found in the one-way ANOVA (HC6).

\section{V.C. Pilot Model Parameters}

The VAF was calculated as a measure for the goodness of fit of the linear pilot model discussed in Section III.D. A VAF of a $100 \%$ indicates that the model was able to perfectly explain all of the variance in the measured pilot control signal $u$. Fig. 9 provides the VAF for all conditions. Values for the VAF ranged between 80 and $85 \%$. These values are similar to values reported in previous experiments. ${ }^{13}$ A significant difference in VAF was introduced by the weighting of the different heave motion components decomposed at the CG. For the conditions that contain full CG pitch heave (C5 and C8), the VAF is significantly lower compared to the conditions with a lower weighting of the CG pitch heave component. The VAF is the lowest for the condition with CG pitch heave only (C5).

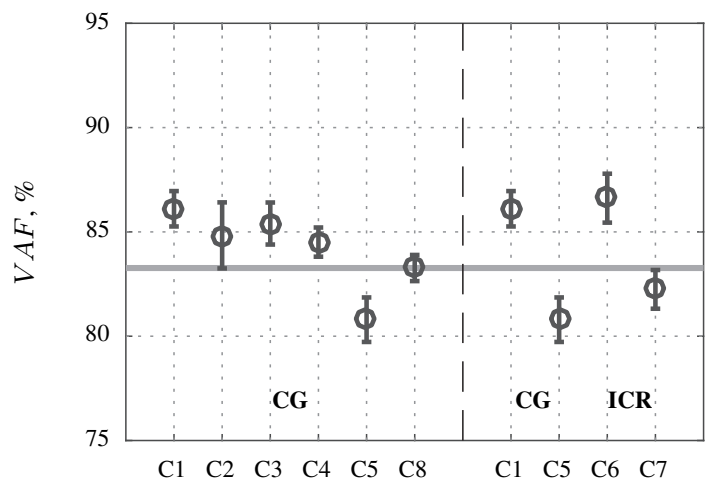

Figure 9. Pilot model variance accounted for.

No significant difference was introduced by the interaction between the decomposition point and the heave component. The decomposition point introduced a significant difference. For the conditions with the heave components decomposed at the ICR, the VAF was higher. The heave component introduced a significant difference that was also reflected in the one-way ANOVA. For the conditions with pitch heave only, the VAF was significantly lower compared to the conditions with CG or ICR heave only. 
The pilot model parameters for each condition are provided in Fig. 10. Figs. 10a and 10b depict the pilot visual and motion gains, respectively. In both gains statistically significant differences were introduced by the weighting of heave motion components decomposed at the CG. Both gains increase with an increase in weighting of the CG pitch heave component. When the percentage of CG pitch heave is higher than the percentage of CG heave, visual and motion gains are similar to the gains in the full-motion condition. For the pilot visual gain, no significant effect from the decomposition point, and no significant interaction between the decomposition point and heave component was found. The heave component introduced a significant difference similar to the one found in the one-way ANOVA. A significant interaction between the decomposition point and the heave component was found for the pilot motion gain. The motion gain in the condition with ICR heave only is higher than the gain in the condition with CG heave only. However, the motion gain in the condition with ICR pitch heave only is similar to the gain in the condition with CG pitch heave only. Finally, the pilot motion gain is lower than the visual gain; however, note that the motion gain estimate also includes aircraft heave and motion washout dynamics (Section III.D).

Fig. 10c depicts the visual lead time constant. The lead time constant was statistically significantly different between motion conditions with different weightings for the heave motion components decomposed at the CG. For an increase in the weighting of the CG pitch heave, a decrease in $T_{L}$ can be observed, indicating a decrease in visual lead generation. Note that the trend is opposite that of the visual gain. When the percentage of CG pitch heave component is higher than that of the CG heave component, the visual lead time constant is similar to the lead time constant in the baseline condition. No significant interaction was found between the decomposition point and the heave component $(\mathrm{DP} 2 \times \mathrm{HC} 2)$. However, the visual lead time constant was found to be significantly lower with the heave components decomposed at the ICR compared to the CG. In addition, $T_{L}$ was significantly lower in the conditions with CG or ICR pitch heave only compared to the conditions with CG or ICR heave only.

The pilot visual time delay is depicted in Fig. 10d. A statistically significant difference between conditions was introduced by the weighting of the heave motion components decomposed at the CG. The visual delay in the baseline condition C8 appeared to be higher compared to the other conditions; however, it was only statistically significantly higher compared to $\mathrm{C} 2$ and $\mathrm{C} 4$. No significant differences were introduced by the interaction between the decomposition point and the heave component, or the factors separately. The pilot motion delay $\tau_{m}$ was statistically significantly affected by the different weightings of the heave motion components decomposed at the CG. The trend in the motion delay across conditions was opposite that of the visual gain. For an increase in the percentage of CG pitch heave and a reduction of CG heave, the motion delay decreased. The motion delay in the conditions with a higher percentage of CG pitch heave than CG heave is most similar to the delay in the baseline condition. No significant interaction between the decomposition point and the heave component was introduced in $\tau_{m}$, and no significant difference from the decomposition point. The heave component introduced the significant difference also found in the one-way ANOVA.

The pilot neuromuscular damping and frequency are depicted in Figs. $10 \mathrm{f}$ and $10 \mathrm{~g}$, respectively. The neuromuscular damping and frequency showed very similar trends across conditions. Both were statistically significantly affected by the different weightings of the heave motion components decomposed with respect to the CG. For an increase in the weighting of the CG pitch heave component, the neuromuscular damping and frequency decreased. Both the neuromuscular damping and frequency in the baseline condition $\mathrm{C} 8$ were most similar to the damping and frequency in the hexapod motion condition C3. Note that condition C3 had an equal weighting of CG pitch heave and CG heave, just like condition $\mathrm{C} 8$. No significant interaction between the decomposition point and the heave component was introduced in the neuromuscular parameters, and no significant difference was introduced by the decomposition point. The heave component introduced a statistically significant effect also found in the one-way ANOVA.

\section{V.D. Crossover Frequencies and Phase Margins}

The crossover frequencies and phase margins of the disturbance and target open loops are depicted in Fig. 11. The different weightings of the heave components decomposed at the CG introduced statistically significant differences in the disturbance crossover frequency $\omega_{d, f_{d}}$. With more emphasis on CG pitch heave (that is, an increase in weighting of the CG heave component), the disturbance crossover frequency increased. The disturbance crossover frequency in conditions with a higher emphasis on CG pitch heave (C4 and C5) was most similar to the baseline condition C8. No significant interaction between the decomposition point and the heave component was introduced, and no significant difference was introduced by the decomposition point. The heave component introduced a significant difference also found in the one-way ANOVA.

The different weightings of the heave motion components decomposed at the CG resulted in statistically significantly different target crossover frequencies between conditions. The observed trend for $\omega_{c, f_{t}}$ is opposite that of $\omega_{c, f_{d}}$; that is, $\omega_{c, f_{t}}$ decreased when the weighting of the CG pitch heave was increased. The target crossover frequency in C3-C5 was most similar to that of C8. The interaction between the heave decomposition point and the heave compo- 

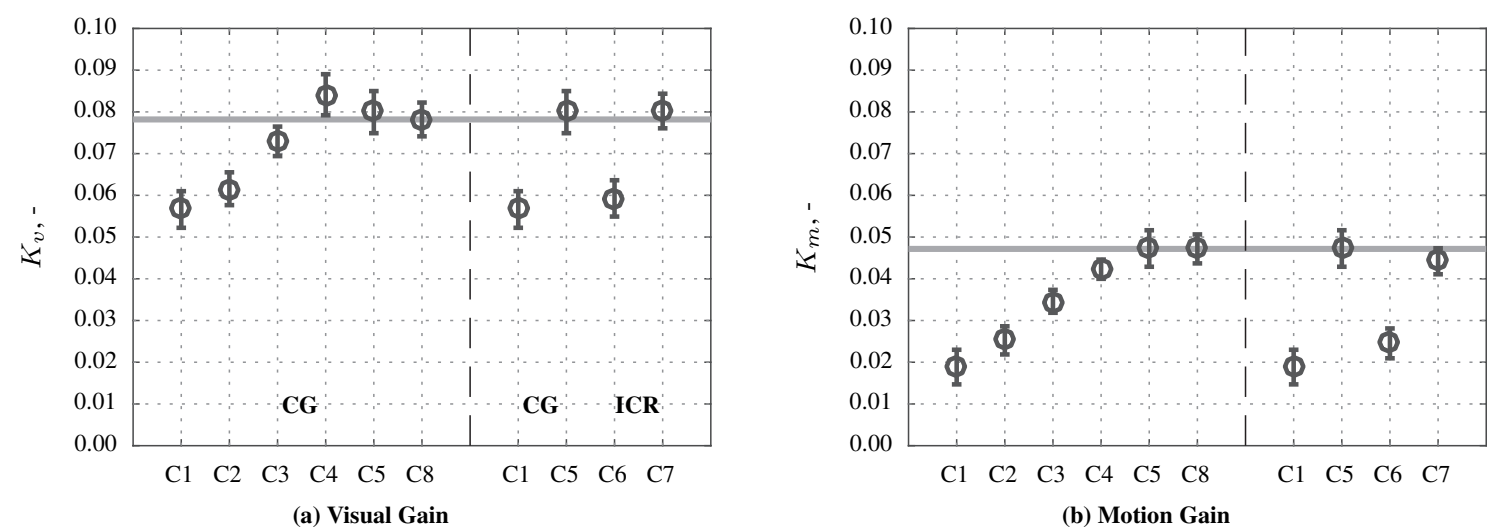

(b) Motion Gain
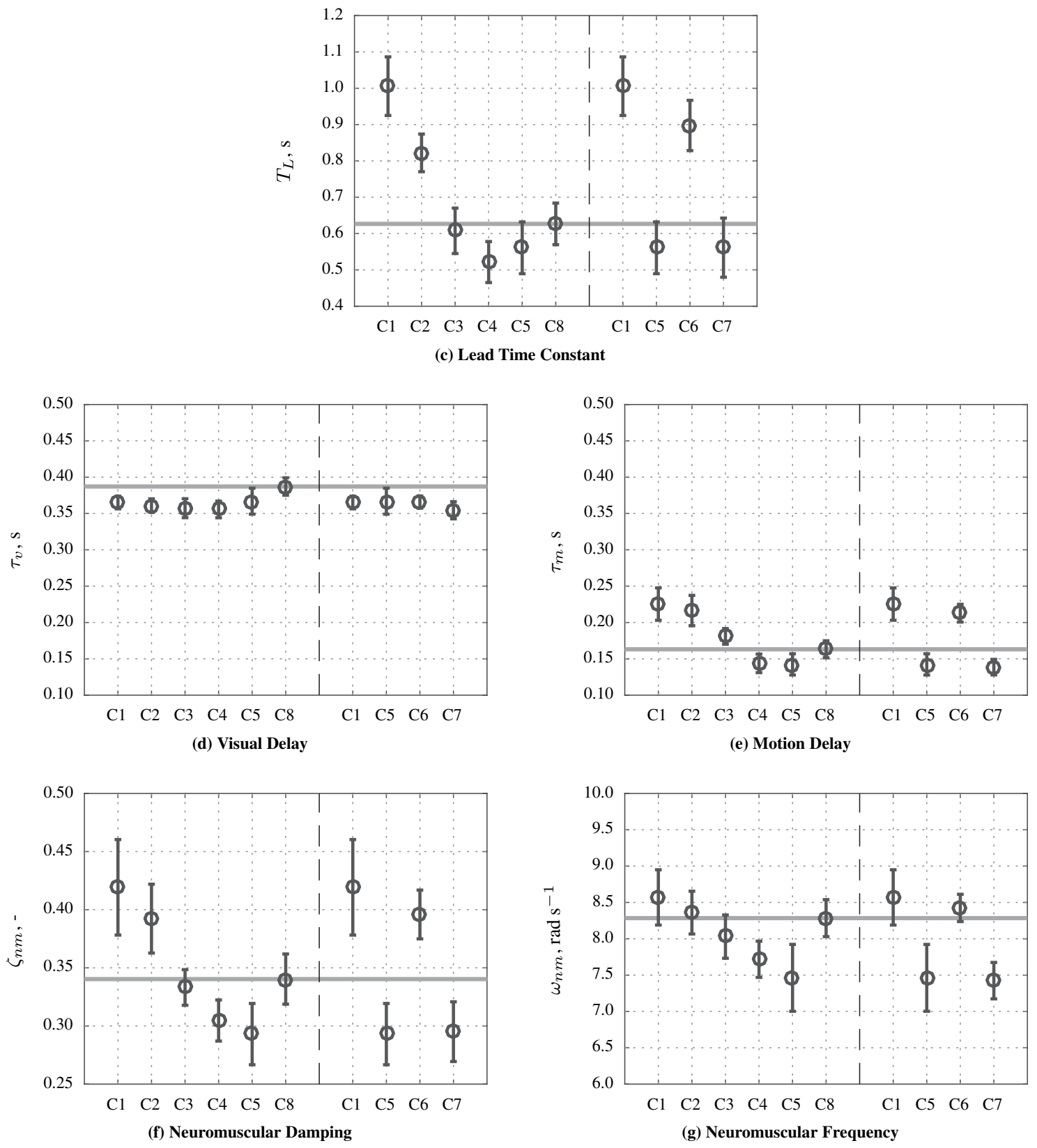

Figure 10. Pilot model parameters. 


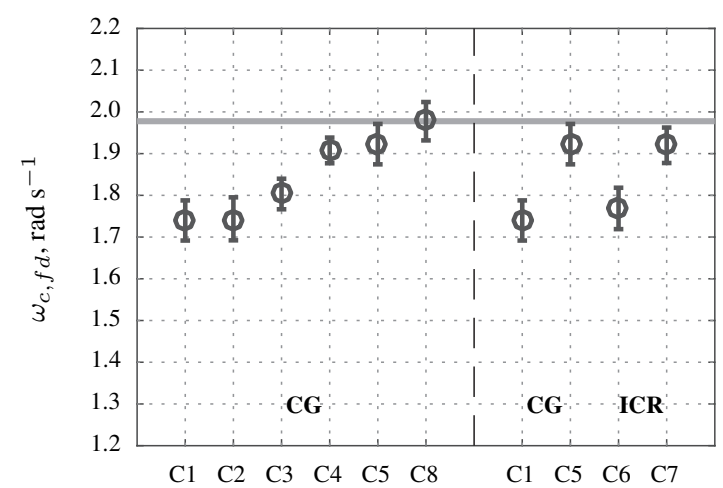

(a) Disturbance Crossover Frequency

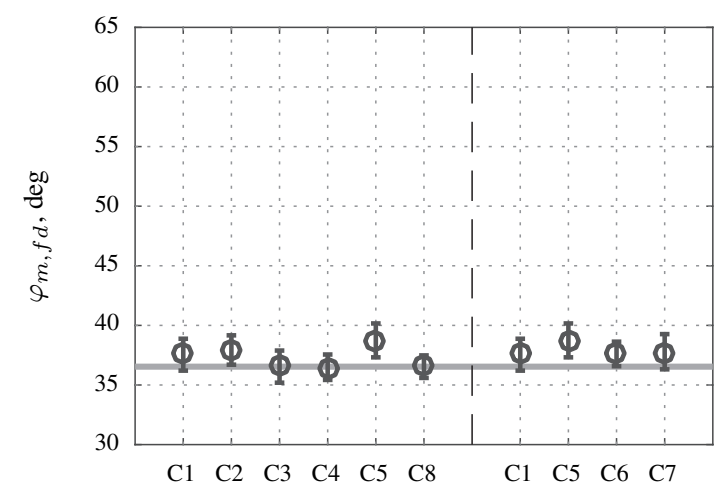

(c) Disturbance Phase Margin

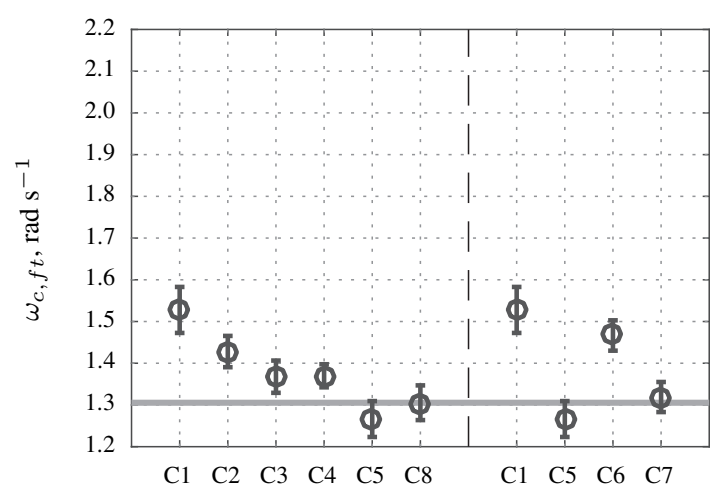

(b) Target Crossover Frequency

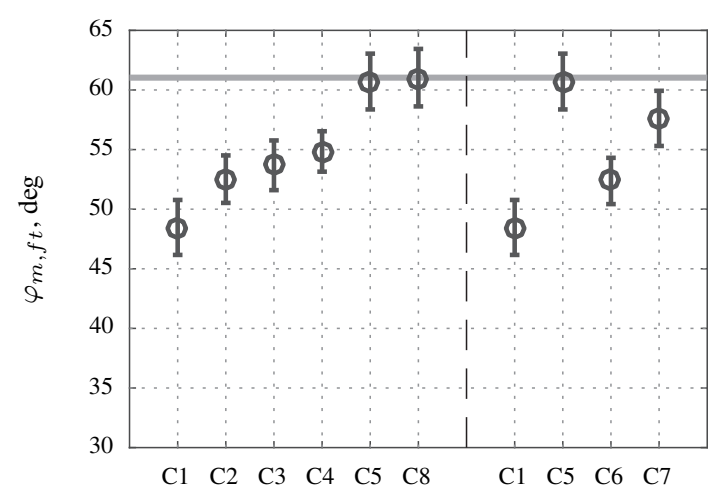

(d) Target Phase Margin

Figure 11. Open-loop parameters.

nent was significant. The target crossover frequency was significantly lower with ICR heave only (C6) compared to CG heave only (C1). However, the target crossover frequency was significantly higher with ICR pitch heave only (C7) compared to CG pitch heave only (C5).

The disturbance phase margin $\varphi_{m, f_{d}}$ was marginally significantly affected by the different weightings of the heave components decomposed at the CG. However, pairwise comparisons with Bonferroni adjustment did not reveal any significant differences between condition pairs. No significant interaction between the decomposition point and heave component was found, or any significant difference introduced by the factor separately.

The target phase margin $\varphi_{m, f_{t}}$ showed an opposite trend that of the target crossover frequency $\omega_{c, f_{t}}$. The target phase margin was statistically significantly affected by the different weightings of the heave components decomposed at the CG. For an increased weighting of CG pitch heave, the phase margin increased. The target phase margin in C5 was closest to the phase margin in C8. Similarly to the target crossover frequency, the interaction between the heave decomposition point and the heave component was significant for the target phase margin. The target phase margin was significantly higher for the condition with ICR heave only compared to the condition with CG heave only. The target phase margin was significantly lower in the condition with ICR pitch heave only compared to the condition with CG pitch heave only.

\section{Discussion}

The VMS experiment discussed in this paper had two objectives. The first objective was to investigate if a higher weighting of the CG pitch heave component compared to the CG heave component, allowing for an increase in motion fidelity of CG pitch heave on a typical hexapod motion system, could result in motion ratings, pilot manual control behavior, and performance closer to what is observed with full aircraft motion. The second objective was to investigate if using the ICR to decompose the heave motion components could result in additional benefits to increase motion fidelity. In addition to heave motion, high-fidelity pitch motion was present in all experimental conditions. 
Pilots rated motion significantly increasingly higher when the weighting of CG pitch heave increased. When the ratio of CG pitch heave to CG heave was higher, ratings were similar to those in the full aircraft motion condition. At the start of the experiment, pilots performed the task with full aircraft motion eight times and were told to rate the motion during the rest of the experiment against this baseline. Due to the nature of the pitch control task, pilots were jolted around considerably more with higher fidelity pitch heave motion. As a result, it was relatively easy to make a distinction between the motion conditions, which resulted in markedly different and consistent ratings in each condition, contrary to some previous investigations with more complex tasks.

Pilot performance was not notably affected by different weightings of the heave components, contrary to previous studies in which the addition of pitch heave motion significantly increased tracking performance. ${ }^{1,2}$ However, the task in Refs. 1 and 2 was more of a disturbance-rejection task, with a disturbance forcing function with four times the variance of the target forcing function. Pilot control activity was statistically significantly affected by the different weightings of heave components. Pilots increased their control activity when the ratio of CG pitch heave to CG heave was higher. This is similar to the findings in Refs. 1 and 2.

Different weightings of the heave motion components decomposed at the CG resulted in significantly different pilot manual control behavior. When the ratio of CG pitch heave to CG heave increased pilot visual and motion gains increased, and the visual lead time constant decreased. This finding is an indication that pilots increasingly relied on simulator motion when the emphasis on CG pitch heave increased, reducing their reliance on visual lead. For the conditions with a higher percentage of CG pitch heave than CG heave, pilot equalization was similar to that in the full-motion condition. These effects are also observed in previous experiments. ${ }^{1,2}$ The pilot visual time delay remained constant across the different hexapod motion conditions (C1-C7); however, was significantly higher for the full motion condition $\mathrm{C} 8$. The pilot motion delay decreased as the ratio of CG pitch heave to CG heave increased. Finally, the neuromuscular damping and frequency both decreased when the ratio of CG pitch heave to CG heave increased. Contrary to the other pilot model parameters, the neuromuscular damping and frequency in the hexapod condition with an equal weighting of CG pitch heave and CG heave was most similar to what is observed in the full aircraft motion condition, which also has an equal weighting of both heave components, but with higher fidelity heave motion. The VAF of the pilot model was constant for the hexapod motion conditions with CG heave (C1-C4). However, the VAF was significantly lower when CG pitch heave was present only (C5). The VAF in the full motion condition was in between the VAF in conditions $\mathrm{C} 1-\mathrm{C} 4$ and $\mathrm{C} 5$. This might indicate that pilot control behavior was less linear in the condition with CG pitch heave only. A possible reason could be that the high-gain pitch heave motion in this condition introduced nonlinear control effects, such as biodynamic feedthrough. The significant differences in pilot control behavior resulted in significant differences in the open-loop characteristics. The disturbance crossover frequency significantly increased, while the target crossover frequency significantly decreased when the ratio of CG pitch heave motion to $\mathrm{CH}$ heave motion increased. The disturbance phase margin remained approximately constant across conditions and the target phase margin increased when the ratio of CG pitch heave motion to CG heave motion increased. These results indicate that pilots were able to increase disturbance-rejection performance while retaining stability margins, and were able to increase target-following stability margins by slightly reducing performance when the level of CG pitch heave increased.

The decomposition point of the heave motion only affected a few dependent measures. Motion was rated significantly higher when the heave was decomposed at the CG compared to the ICR, most likely due to the higher intensity of the CG pitch heave motion compared to the ICR pitch heave motion. The pilot motion gain was significantly lower for the ICR pitch heave and significantly higher for the ICR heave compared to their CG counterparts. The visual lead time constant was significantly lower when the heave was decomposed at the ICR and the pilot model VAF was significantly higher. Finally, the target crossover frequency for the ICR pitch heave was significantly lower and the target crossover frequency for the ICR heave significantly higher compared to the same heave components decomposed at the CG. For the target phase margin, a similar but opposite significant interaction was found. These results indicate that the lower intensity ICR pitch heave compared to CG pitch heave increased target-following performance and decreased stability margins. The opposite was true for the ICR heave compared to the CG heave, most likely due to fact that the CG heave still contains a small but opposite pitch heave component that is not present in the ICR heave (Section II).

It should be noted here that the pilot model used in this study introduced a modeling error by lumping together the perception of rotational pitch motion and translational heave motion through the semicircular canals and otoliths, respectively. In addition, the pilot model responses contained aircraft heave dynamics and simulator motion dynamics. These types of modeling errors are a result of the fact that only two pilot response channels can be identified using the technique used here. The result is that variations in the pilot motion gain and motion delay are not solely due to a 
change in pilot control strategy. However, variations in remaining pilot model parameters and open-loop characteristics are still accurate and reveal the significant effects of the heave component ratios on pilot manual control behavior.

The results presented in this paper indicate that it might be possible to improve motion cueing in hexapod flight simulators to induce pilot control behavior and performance more similar to what is observed in real flight by weighting different components of the aircraft motion differently, allowing for an increase in motion fidelity of the motion components that are most important for a specific task. The task in this paper was a pitch attitude control task and hence putting more emphasis on the pitch heave component of the total pilot station heave and increasing its fidelity resulted in pilot control behavior and performance similar to that with full aircraft motion. This motion cueing strategy could be applied for real flight tasks that are similar in nature, such as manually following a glideslope in an approach or regulating pitch attitude in a stall recovery. For different tasks, other motion components might be more important; for example, $C G$ heave is more important when regulating the flare during a landing. This means that if these motion cueing strategies are to be applied in flight simulation, different motion logic settings would be required for different parts of a flight. How this could be implemented in current flight simulator is beyond the scope of this paper.

\section{Conclusions}

Pilots performed a pitch attitude control task in the Vertical Motion Simulator with pitch motion and different weightings of heave motion components decomposed at the aircraft's center of gravity or instantaneous center of rotation. The motion conditions were tuned to fit in a typical hexapod motion space. Pilots also performed the task in a baseline full-motion condition that provided real aircraft motion cues. When the ratio of center of gravity pitch heave to center of gravity heave increased in the hexapod conditions, allowing for increased overall motion gains and reduced washout break frequencies, pilot manual control behavior and performance became increasingly more similar to what is observed with full aircraft motion. Pilot visual and motion gains significantly increased, while visual lead generation decreased. The pilot visual time delay remained approximately constant and the pilot motion time delay decreased. The neuromuscular damping and frequency both decreased, with their values more similar to what is observed with real aircraft motion when there was an equal weighting of center of gravity pitch heave and center of gravity heave motion. In terms of open-loop performance, the disturbance and target crossover frequency increased and decreased, respectively, and their corresponding phase margins remained constant and increased, respectively. The decomposition point of the heave components only had minor effects on pilot manual control behavior and performance. The results presented here suggest that improved motion cueing on hexapod motion platforms might be possible for certain flight tasks by differently weighting the heave components decomposed at the aircraft's center of gravity.

\section{Appendix}

Table 3 provides the results of the ANOVAs performed on the dependent measures of the experiment. The results of the one-way ANOVA with conditions C1-C5 and C8 are given inTable 3a. The results of the two-way ANOVA with conditions $\mathrm{C} 1, \mathrm{C} 5, \mathrm{C} 6$, and $\mathrm{C} 7$ are given in Table 3b. As part of the analysis, checks for outliers, normal distribution, and homogeneity of variances were performed. Normality of the data was assessed by Shapiro-Wilk's test of normality. Homogeneity of variances was assessed by Levene's test of equality of variances. As dependent measures contained only few outliers and were non-normally distributed in only a few conditions, no corrections were applied to the data. Whenever the assumption of homogeneity of variances was violated, a Greenhouse-Geisser correction was used for the degrees of freedom of the F-distribution.

\section{Acknowledgments}

The authors thank everybody at NASA Ames SimLabs who contributed to the experiment. We especially thank Emily Lewis and Steve Norris for their valuable contributions.

\section{References}

${ }^{1}$ Zaal, P. M. T., Pool, D. M., de Bruin, J., Mulder, M., and van Paassen, M. M., "Use of Pitch and Heave Motion Cues in a Pitch Control Task," Journal of Guidance, Control, and Dynamics, Vol. 32, No. 2, March-April 2009, pp. 366-377.

${ }^{2}$ Pool, D. M., Zaal, P. M. T., van Paassen, M. M., and Mulder, M., "Effects of Heave Washout Settings in Aircraft Pitch Disturbance Rejection," Journal of Guidance, Control, and Dynamics, Vol. 33, No. 1, Jan.-Feb. 2010, pp. 29-41. 
Table 3. Analysis of variance results.

(a) Effects of heave component ratio (C1-C5,C8).

\begin{tabular}{cccc}
\hline \hline \multirow{2}{*}{ dependent } & & \multicolumn{2}{c}{ HC6 } \\
\cline { 3 - 4 } measure & $d f$ & $F$ & Sig. \\
\hline$m r$ & $1.403,25.245^{g g}$ & 40.114 & $* *$ \\
\hline$R M S_{e}$ & $2.979,53.622^{g g}$ & 2.355 & $*$ \\
$R M S_{u}$ & $5.0,90.0$ & 11.828 & $* *$ \\
\hline$V A F$ & $2.858,51.448^{g g}$ & 13.198 & $* *$ \\
$K_{v}$ & $5.0,90.0$ & 23.700 & $* *$ \\
$K_{m}$ & $2.198,39.555^{g g}$ & 43.179 & $* *$ \\
$T_{L}$ & $5.0,90.0$ & 31.581 & $* *$ \\
$\tau_{v}$ & $3.219,57.947^{g g}$ & 3.157 & $* *$ \\
$\tau_{m}$ & $3.067,55.201^{g g}$ & 18.379 & $* *$ \\
$\zeta_{n m}$ & $2.518,45.326^{g g}$ & 12.910 & $* *$ \\
$\omega_{n m}$ & $5.0,90.0$ & 5.951 & $* *$ \\
\hline$\omega_{c, d}$ & $5.0,90.0$ & 19.585 & $* *$ \\
$\phi_{m, d}$ & $5.0,90.0$ & 2.189 & $*$ \\
$\omega_{c, t}$ & $2.664,47.946^{g g}$ & 18.794 & $* *$ \\
$\phi_{m, t}$ & $3.415,61.471^{g g}$ & 19.588 & $* *$ \\
\hline \hline
\end{tabular}

$$
\begin{aligned}
* * & =\text { significant }(p<0.05) \\
* & =\text { marginally significant }(0.05 \leq p<0.1) \\
- & =\text { not significant }(p \geq 0.1) \\
g g & =\text { Greenhouse-Geisser sphericity correction }
\end{aligned}
$$

${ }^{3}$ Zaal, P. M. T., Pool, D. M., van Paassen, M. M., and Mulder, M., "Comparing Multimodal Pilot Pitch Control Behavior Between Simulated and Real Flight," Journal of Guidance, Control, and Dynamics, Vol. 35, No. 5, Sept. - Oct. 2012, pp. 1456-1471.

${ }^{4}$ van Wieringen, A. T., Pool, D. M., van Paassen, M. M., and Mulder, M., "Effects of Heave Washout Filtering on Motion Fidelity and Pilot Control Behavior for a Large Commercial Airliner," Proceedings of the AIAA Modeling and Simulation Technologies Conference, Portland (OR), No. AIAA-2011-6323, 8-11 Aug. 2011.

${ }^{5}$ McRuer, D. T., Graham, D., Krendel, E. S., and Reisener, W., "Human Pilot Dynamics in Compensatory Systems. Theory, Models and Experiments With Controlled Element and Forcing Function Variations," Tech. Rep. AFFDL-TR-65-15, Wright Patterson AFB (OH): Air Force Flight Dynamics Laboratory, 1965.

${ }^{6}$ Stapleford, R. L., Peters, R. A., and Alex, F. R., "Experiments and a Model for Pilot Dynamics with Visual and Motion Inputs," Tech. Rep. NASA CR-1325, NASA, 1969.

${ }^{7}$ Jex, H. R. and Magdaleno, R. E., "Roll Tracking Effects of G-vector Tilt and Various Types of Motion Washout," Fourteenth Annual Conference on Manual Control, University of Southern California, Los Angeles (CA), 25-27 April 1978, pp. 463-502.

${ }^{8}$ Zaal, P. M., Pool, D. M., Mulder, M., van Paassen, M. M., and Mulder, J. A., "Identification of Multimodal Pilot Control Behavior in Real Flight," Journal of Guidance, Control, and Dynamics, Vol. 33, No. 5, Sept.-Oct. 2010, pp. 1527-1538.

${ }^{9}$ Pool, D. M., Zaal, P. M. T., Damveld, H. J., and van Paassen, M. M. M. M., "Evaluating Simulator Motion Fidelity using In-Flight and Simulator Measurements of Roll Tracking Behavior," Proceedings of the AIAA Modeling and Simulation Technologies Conference, Minneapolis (MN), No. AIAA-2012-4635, 13-16 Aug. 2012.

${ }^{10}$ Damveld, H. J., Beerens, G. C., van Paassen, M. M., and Mulder, M., "Design of Forcing Functions for the Identification of Human Control Behavior," Journal of Guidance, Control, and Dynamics, Vol. 33, No. 4, July - Aug. 2010, pp. 1064-1081.

${ }^{11}$ Danek, G. L., "Vertical Motion Simulator Familiarization Guide," NASA Technical Memorandum NASA TM-103923, NASA, 1993.

${ }^{12}$ Beard, S. D., Reardon, S. E., Tobias, E. L., and Aponso, B. L., "Simulation System Optimization for Rotorcraft Research on the Vertical Motion Simulator," Proceedings of the AIAA Modeling and Simulation Technologies Conference, Minneapolis (MN), No. AIAA-2012-4634, 1316 Aug. 2012.

${ }^{13}$ Zaal, P. M. T., Pool, D. M., Chu, Q. P., van Paassen, M. M., Mulder, M., and Mulder, J. A., "Modeling Human Multimodal Perception and Control Using Genetic Maximum Likelihood Estimation," Journal of Guidance, Control, and Dynamics, Vol. 32, No. 4, July-Aug. 2009, pp. 1089-1099.

${ }^{14}$ Zaal, P. M. T., Popovici, A., and Zavala, M. A., "Effects of False Tilt Cues on the Training of Manual Roll Control Skills," Proceedings of the AIAA Modeling and Simulation Technologies Conference, Kissimmee, Florida FL, No. AIAA-2015-0655, 5-9 Jan. 2015. 